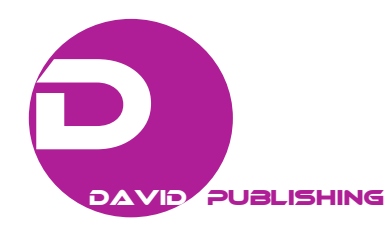

\title{
Real-Time, Automatic and Wireless Bridge Monitoring System Based on MEMS Technology
}

\author{
Bo Qiu ${ }^{1}$ and Jianda $\operatorname{Tan}^{2}$ \\ 1. Sun Engineering Consultants International, Inc., Guangzhou 510620, China \\ 2. Mechanical and Aerospace Engineering Department, University of California, San Diego, CA 92092, USA
}

\begin{abstract}
Currently, the monitoring of bridges in China heavily relies on manual operation, which has several major problems. It generally takes a very long time to complete an inspection process on bridges. The manual data is sometimes unreliable or even wrong in the case of careless operation. The inspection activity itself is dangerous for inspectors, e.g., bridges are located in the sea or river. Some semi-automatic monitoring methods are recently employed, but they are either very expensive or do not work properly. Therefore, the traditional bridge monitoring process becomes an increasing challenge for bridge operators. In this paper, a real-time and automatic bridge monitoring system is presented to meet the bridge monitoring needs, and MEMS (Micro Electro Mechanical Systems) are the key building block in this system. By using the MEMS-based sensors, it is much more efficient and accurate in monitoring bridges with the measurement of inclination, acceleration, displacement, moisture, temperature, stress and other data.
\end{abstract}

Key words: Bridge monitoring system, MEMS (Micro Electro Mechanical Systems), automatic, real-time, wireless.

\section{Introduction}

Bridge monitoring system has been an active research area in recent years. In general, the purpose of bridge monitoring is the early detection and characterization of damage conditions before a threat is posed to the structural integrity of a bridge. Similar to human body, a bridge, if diagnosed properly, could go through early treatment to restore its health.

The current state of the aging bridge infrastructure in China demands more reliable diagnostic mechanism for a large amount of bridges. Traditional bridge monitoring methods entail an annual manual operation that relies on visual inspection and temporary installation of devices. This mechanism is obviously subjective by nature, and it is almost unavoidable to introduce inaccuracies and even errors [1].

This paper presents a remote, wireless and automatic bridge monitoring system, which mainly includes the following sensors: MEMS (Micro Electro

Corresponding author: Jianda Tan, Ph.D., research field: bridge monitoring system.
Mechanical Systems)-based inclinometer, MEMS-accelerometer, strain gauge, displacement meter, temperature and humidity sensor and cable force gauge. All of these sensors are connected to its own signal acquisition and wireless transmission module. Those data collected will be processed by bridge health monitoring software which analyzes the data and reports any warning and emergency conditions.

It is noted that the major distinction between this presented monitoring system and traditional method is the usage of MEMS sensors, which are capable of providing real-time data and make instant evaluation of bridges. Compared with the counterparts, MEMS sensors are much cheaper and more reliable as well.

There are four major parts in the MEMS-based bridges monitoring system: the sensor installation, data processing, health monitoring software development and power management. In the following part, the general design of the monitoring system will be firstly introduced, and then four main components in this developed system will be presented. 


\section{General Design of the MEMS-Based} Monitoring System

Fig. 1 shows the flow chart on the basic framework of MEMS-based monitoring system. The sensors are classified into three main kinds: MEMS inclinometer, MEMS accelerometer and other related sensors. The basic idea is that after collecting all the data from different sensors, the software calculates and displays inclination, vibration and other parameters which reflect the operating condition of bridges. It is mentioned that combined with the empirical correlations and engineering calculation, the key bridge dynamic properties like the variation of stiffness and natural frequency can be estimated based on the data from MEMS-accelerometer, therefore, the health condition and overall status of the bridge can be evaluated.

\section{Installation}

The MEME inclinometer should be installed in bridge tower, deck and bridge pier so that inclination along and across the bridge is measured. Inclinometer is arranged along the deck by a fixed distance so that deflection as a function of position can be derived. The accuracy of the deflection function is positively related to the density of the inclinometer. The accuracy of the inclinometer itself could reach $0.005^{\circ}$ [2].

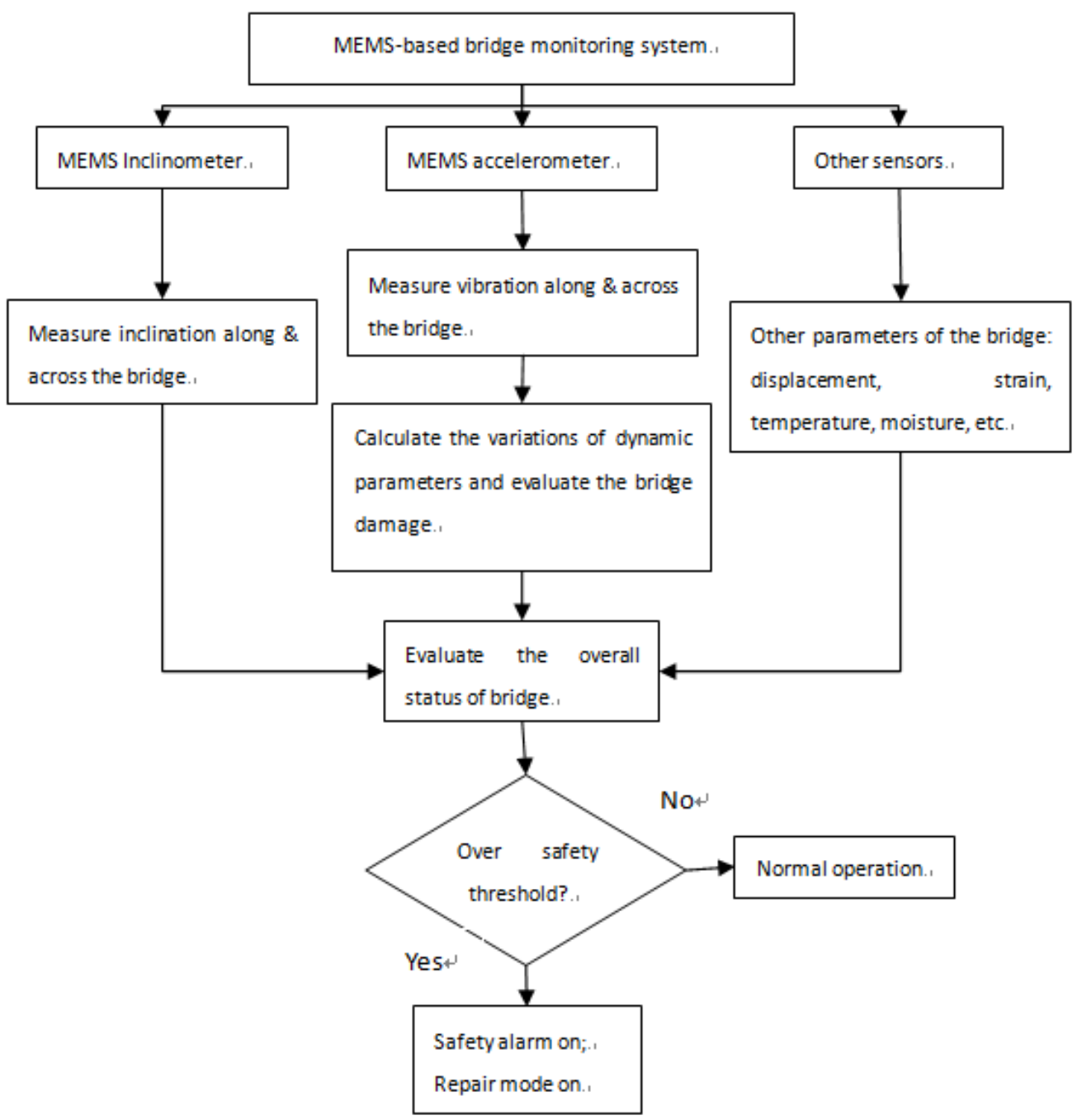

Fig. 1 The basic framework of MEMS-based bridge monitoring system. 


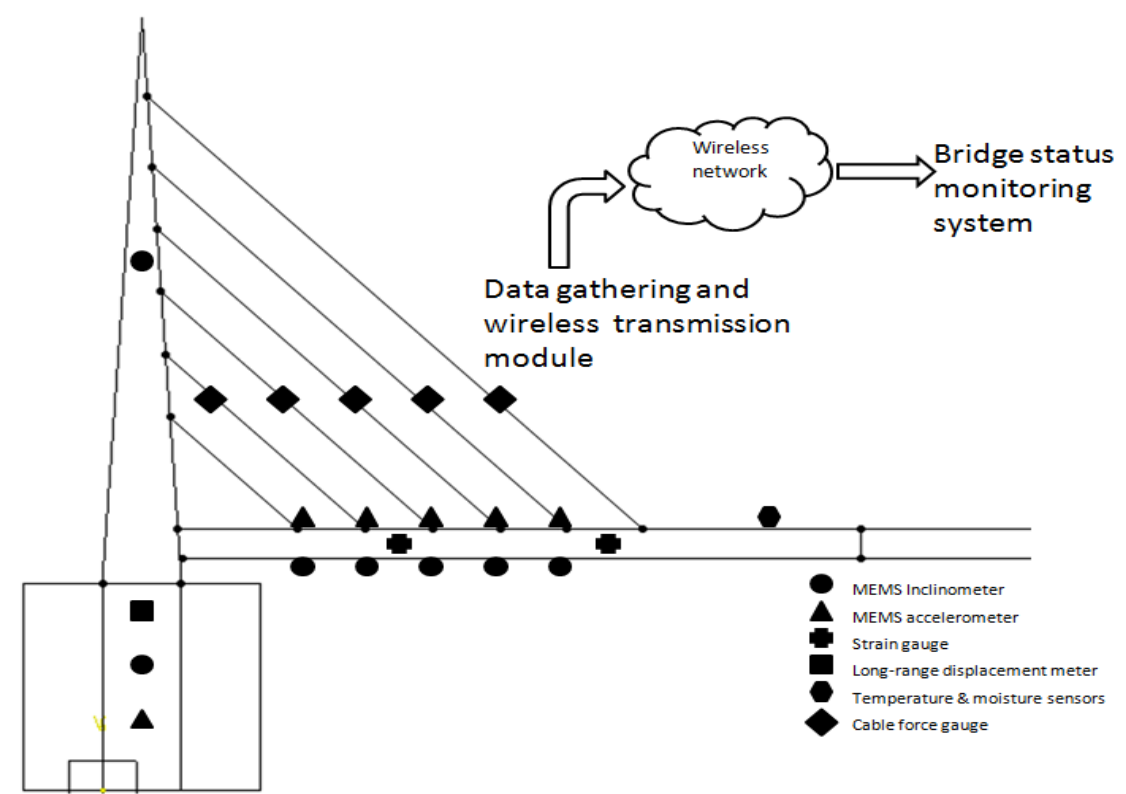

Fig. 2 The general sensor installation plan on a bridge.

The MEMS accelerometer should be installed in the deck and bridge pier. The acceleration along the bridge and across can be measured. Velocity and displacement could be obtained by integrations of acceleration. Considering the drifting phenomenon could affect or even dominate the integration process [3]. A self-compensation algorithm based on zero acceleration without loading is developed to eliminate the drifting of the accelerometer [4]. The dynamic deflection and natural frequency of the bridge can be derived based on acceleration measurement. The acceleration data can also be a source for evaluating the overall performance of a bridge and damage control.

The strain gauge is installed inside the bridge pier to monitor strain in the concrete and steel.

Cable force gauge is installed in the cable of bridge (in the case of cable-stayed bridge) to measure tensile force. Long-range displacement meter is installed on the surface of the bridge pier to monitor the settlement of the pier. Temperature and moisture sensors are installed in the bridge parts which are sensitive to the variation of environment.

Additional sensors can be installed as well to meet more specific need of certain type of bridges, for example, wind sensors can be employed to detect the strength of wind. Fig. 2 outlines the general sensor installation plan on a bridge.

\section{Data Processing: Compensation, Filtering and Localization}

The major task of data processing is the development of the embedded program loaded on the wireless sensor. Data measurement could shift because of temperature variation. The relationship between the measurement drifting and temperature could be obtained by applying least square technique on result from calibration experiment. A third-order polynomial function should be a good-enough model of temperature drifting compensation. Temperature compensation should be applied individually to various types of sensors because each type of sensors differs in its drifting properties.

LMS (least mean squares) algorithm could be used to filter raw data from sensors. For MEMS accelerometer and inclinometer, there is no need to filter out high frequency response since that is critical for natural frequency derivation. For strain gauge and cable-force gauge, however, a low-pass filter should be used to filter out high frequency noise and get cleaner data for evaluation purpose.

It should be noted that there is a need to localize 
part of the computation in the micro-controller especially in the event-triggered case.

The details of the micro-controller, wireless transmission module and power circuit board are not described here. This article mainly focuses on the application of MEMS technology in bridge monitoring.

\section{Health Monitoring Software Development}

The bridge health monitoring software is developed by the following five design components or considerations:

- Configure the parameters of the serial port and initialize;

- Data gathering, summarizing and storing;

- Transform the measurement data into corresponding value and calculate the desired parameters according to the theories of bridge engineering and the comparison of history data;

- Display the data in 2D/3D curve or animations and deposit the data into files;

- When certain values are above threshold, the warning information is automatically sent to the bridge operators by text, email, etc.

\section{Power Management}

To overcome the economic challenge in the practical field implementation, the overall energy consumption of this system needs to be reduced. Economics consideration is the main factor that impedes the transition of sensor-based technique from research facility to practical field $[5,6]$. Power supply is one of the major concerns.

Regardless of power source, there are two major mechanisms to control the wireless bridge monitoring system: time-trigger and event-trigger.

In time-trigger mechanism, the system switches between sleeping mode and working mode at different time. In this mechanism, the monitoring system only records and transmits a portion of data. It enables the system to sleep most of the time and eliminates most of the communication.

In event-trigger approach, the system only records and transmits data when vibration exceeds a predefined threshold. In the application of bridge, this approach would effectively filter out low frequency components in the data, thereby making some types of damages invisible to the observer.

Both power management modes will be available to user and the default setting is time-trigger. Another advantage of active power management is that it extends the lifespan of these electronic devices because they are "off" most of the time.

\section{Challenges}

Real-time and automatic bridge monitoring system has many advantages over the traditional method. However, some concerns have to be addressed. The specific location to install various sensors could vary depending on different situations. For example, the strain gauge is installed inside the bridge pier which means that the installations happen simultaneously with the construction of the bridge. Sensor damage may occur during the bridge construction. Also, the way that strain gauge is connected to its transmission module and battery source differs depending on the bridge type [7]. Secondly, a bridge is usually designed to last for 100 years while MEMS sensors have a much shorter life circle. There is a need to address the deterioration of sensors and replace them when necessary. The monitoring system should be designed in a way that makes MEMS sensors easily replaceable.

\section{Conclusions}

In this paper, the advantages and challenges of real-time and automatic bridge monitoring system based on MEMS technique over the traditional way of manual operation are introduced. The general design and major parts of the MEMS-based monitoring system is also presented. The system still needs more customization to meet specific requirements of bridge operators. Further improvement on this system will be 
carried out in the near future.

\section{References}

[1] Wenzel, H. 2009. Front Matter. New Jersey: John Wiley \& Sons, Ltd.

[2] Kulchin, Y., Vitrik, O., Dyshluk, A., Anochin, P., and Ilin, A. 2004. "Health Monitoring on Engineering Structures on the Basis of Fiber Optic Tilt Sensor." Fundamental Problems of Optolectronics and Microelectronics II: 288-94.

[3] Park, H. S., Lee, H. M., Adeli, H., and Lee, I. 2007. “A New Approach for Health Monitoring of Structures: Terrestrial Laser Scanning." Computer-Aided Civil and Infrastructure Engineering 22 (1): 19-30.
[4] Vaccaro, R., Gindy, M., Nassif, H., and Velde, J. 2006. "An Algorithm for Estimating Bridge Deflection from Accelerometer Measurements." In Conference Record-Asilomar Conference on Signals, Systems and Computers, 540-4.

[5] Farhey, D. N. 2005. "Bridge Instrumentation and Monitoring for Structural Diagnostics.” Structural Health Monitoring 4 (4): 301-18.

[6] Farhey, D. N. 2007. "Quantitative Assessment and Forecast for Structurally Deficient Bridge Diagnostics." Structural Health Monitoring 6 (1): 39-48.

[7] Farrar, C. R., and Worden, K. 2007. "An Introduction to Structural Health Monitoring." Phil. Trans. R. Soc. A 365: 303-15. 